\title{
MINERAL OCCURRENCES AND INDICATORS IN THE BAIRD MOUNTAINS QUADRANGLE, NORTHWESTERN ALASKA
}

\author{
By Jeanine M. Schmidt and Gayle L. Allegro
}

\section{INTRODUCTION}

This compilation of mineral occurrences and indicators is a product of the Alaskan Mineral Resource Assessment Program (AMRAP) Level III mapping project within the Baird Mountains $1^{\circ}$ by $3^{\circ}$ quadrangle during the $1983-86$ field seasons. This report consists of a sample-locality map and table that summarizes previously known mineral occurrences and describes new occurrences and mineralization indicators located during the AMRAP field program. Sample locations on the map are numbered in two groups (1-194 and 195-290), both increasing from upper left to lower right, by 1:63,360-scale quadrangle (D-6, D-5, D-4 ... D-1, C-6, C-5 ... C-1 ...). Map numbers 1-194 show locations of sulfide and oxide minerals, barite, fluorite, and copper carbonate and copper oxide minerals. The numbers also include locations of rock samples described in the field as gossans-bleached, altered-appearing, or heavily ironstained rocks that have high geochemical values in one or more elements (greater than one standard deviation above background). Most macroscopically visible mineral occurrences are noted, including previously published occurrences $(\mathrm{P})$, minerals identified in hand sample $(\mathrm{H})$, and minerals found in panned concentrates of stream sediments and in leached residues of carbonate rocks (M). Trace to minor amounts of iron sulfides in greenstones and metabasalts are not tabulated, since these are a normal accessory phase of mafic lithologies and not necessarily indicative of mineralization. Map numbers 195-290 show the locations of placer gold deposits (G), as well as creeks (C), and rocks or quartz boulders $(F)$ noted in the field to be distinctly red or iron stained. The accompanying table includes the longitude, latitude, and 15-minute quadrangle for each sample site, as well as sample numbers, sample types, name, if any, and short geologic descriptions. Geologic descriptions are quoted from individual field geologists' notes. Where available, the mineralogy, selected geochemical values, and published references for each occurrence are also listed. Sample numbers in the table have been shortened by omitting the " 8 " that precedes all samples collected in the years $1983-86$ (86MZ024 is listed as 6MZ024).

\section{BACKGROUND}

The Baird Mountains quadrangle has historically been uninhabited and is geographically isolated. It lies east and south of the Noatak River and north of the Kobuk River, which are the main transportation routes from the coast into the western Brooks Range. During and after the Nome gold rush at the turn of the century, a few prospectcrs established temporary (one to two winters) camps along the Kobuk river, and some ventured up the lower portions of the Squirrel River. Gold was discovered in the Klery Creek area in 1909 and was mined intermittently until the early 1940's. The most consistent gold production was fron Klery Creek itself, where hand-panning was supplemented with dragline, hydraulic, and dredge production (Cobb, 1975). Most of the Baird Mountains, however, were apparently' never prospected.

A 1945 report by Eskil Anderson of the Tenitory of Alaska Department of Mines (unpub. data) repots a possible cassiterite discovery by prospector Peter Wonds in the Eli River area in 1935. Letters in 1955 from lawyers to the heirs of the claim suggest that the occurrence may have been located in the Noatak quadrangle west of the area of this report. Tin minerals have not been identified in the AMRAP program anywhere in the Baird Mo intains quadrangle. Bear Creek Mining Company conducted very broadscale regional reconnaissance stream-sediment sampling and mapping in the western Brooks Range in the late 1950's and early 1960's. Their followup work led to staking and drilling of the Omar claims ( $\mathrm{B}-4$ and $\mathrm{C}-4$ quadrangles) in the early to middle 1960 's. These claims were subsequently dropped. There was renewed activity on the Timber Creek gold placers in the mid-1980's, but it is not known if this was development work or mining/production.

In 1978, most of the quadrangle was incorporated by Presidential proclamation into the Noatak National Monument and Kobuk Valley National Monument, portions of which are designated wilderness areas, and all of which are closed to mineral entry. The "D-1" status lands of the Squirrel River basin are presently administered by the Bureau of Land Management and are under consideration for inclusion into the National Park system as a designated Wild and Scenic River. Some Native-owned lands are located in the southern and southeastern parts of the quadrangle.

In summary, the geology of the Baird Mountains quadrangle has never been effectively evaluated by prospectors, government agencies, or mineral exploration companies. In part to counteract this long history of neglect, this report in- 
cludes all mineral occurrences identified during mapping that could possibly be related to mineralization. The number of occurrences and indicators found during the course of a reconnaissance geologic mapping and drainage-sampling program suggests that the absence of previously known mineral prospects or mines in the quadrangle is a function of its exploration history and does not reflect its true mineral resource potential.

\section{ACKNOWLEDGMENTS}

The following geologists and assistants have contributed field observations and samples to this compilation: Peter Folger (samples numbered 3PF-5PF, collected in 1983 through 1985), Jeanine Schmidt (3JS-6JS), Susan Karl (3SK-6SK), Inyo Ellersieck (3EK-4EK), Bill Thompson (4BT-5BT), Randy Baker (4RB), Michael Flaherty (5MF), Julie Dumoulin (4DN-5DN), Mark Zayatz (6MZ), and Alison Till (5TI-6TI). Kennecott Exploration Co. (formerly Bear Creek Mining Co., and presently BP Minerals America), provided information on drilling results at the Omar prospect. Thanks are also due to pilots Charlie Jones, Bill Bolling, Will Curry, Dave Blair, and Paul Walters, for their flying support of the sampling programs.

\section{REFERENCES CITED}

Brosgé, W.P., Reiser, H.N., and Tailleur, I.L., 1967, Copper analyses of selected samples, southwestern Brooks Range, Alaska: U.S. Geological Survey open-file report.

Cobb, E.H., 1972, Metallic mineral resources map of the Baird Mountains quadrangle, Alaska: U.S. Geological Survey Miscellaneous Field Studies Map MF-386, scale 1:250,000.

1975, Summary of references to míneral occurrences (other than mineral fuels and construction materials) in northern
Alaska: U.S. Geological Survey Open-File Report 75-628, $106 \mathrm{p}$.

Cobb, E.H., Mayfield, C.F., and Brosgé, W.P., 1981, Summaries of data on and lists of references to metallic and selected nonmetallic mineral occurrences in eleven quadrangles in northern Alaska, Supplement to Open-File Report 75-628: U.S. Geological Survey Open-File Report 81-767, 38 p.

Degenhart, C.E., Griffis, R.J., McQuat, J.F., and Bigelow, C.G., 1978, Mineral studies of the westem Brooks Range performed under contract to the U.S. Bureau of Mines, Contract \#J0155089: U.S. Bureau of Mines Open-File Report 103-78, $529 \mathrm{p}$.

Folger, P.F., and Schmidt, J.M., 1986, Geology of the carbonatehosted Omar copper prospect, Baird Mountains, Alaska: Economic Geology. v. 81, no. 7, p. 1690-1695.

Karl, S.M., Schmidt, J.M., and Folger, P.F., 1985, Selected anomalous rock and sediment samples from central and northwestern Baird Mountains quadrangle, in BartschWinkler, Susan, ed., The United States Geological Survey in Alaska: Accomplishments during 1984: U.S. Geological Survey Circular 967, p. 8-13.

Schmidt, J.M., and Folger, P.F., 1986, Pb-Zn-Ag mineralization in Paleozoic dolostones, Powdermilk prospect, Baird Mountains B-4 quadrangle, in Bartsch-Winkler, Susan and Reed, K.M., eds., Geologic Studies in Alaska by the U.S. Geological Survey during 1985: U.S. Geological Survey Circular 978, p. 1921.

U.S. Bureau of Mines Staff, Field Operations Center, Juneau, 1978, Mineral data appraisal of the proposed Noatak national ecological preserve, Alaska: A preliminary comment: U.S. Bureau of Mines Open-File Report 67-78, 22 p.

Zayatz, M.R., Thompson, W.B., Bailey, E.A., Sutley, S.J., Folger, P.F., Karl, S.M., and Schmidt, J.M., 1988, Analytical results and sample locality maps of mineralized and unmineralized rock samples from the Baird Mountains quadrangle, Alaska: U.S. Geological Survey Open-File Report 88-256-A, scale $1: 250,000,159 \mathrm{p}$. 


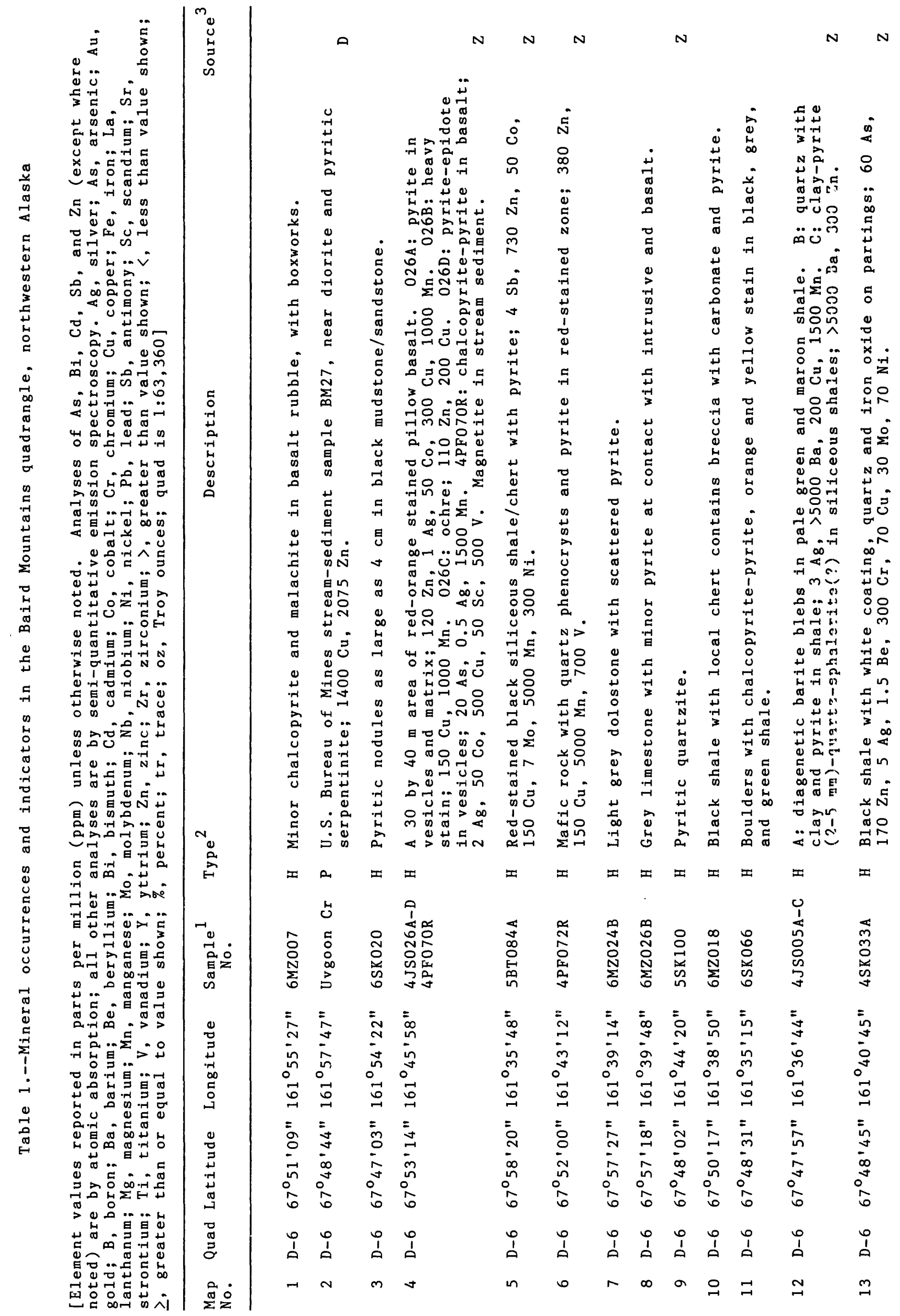




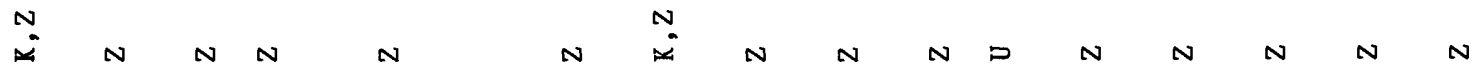

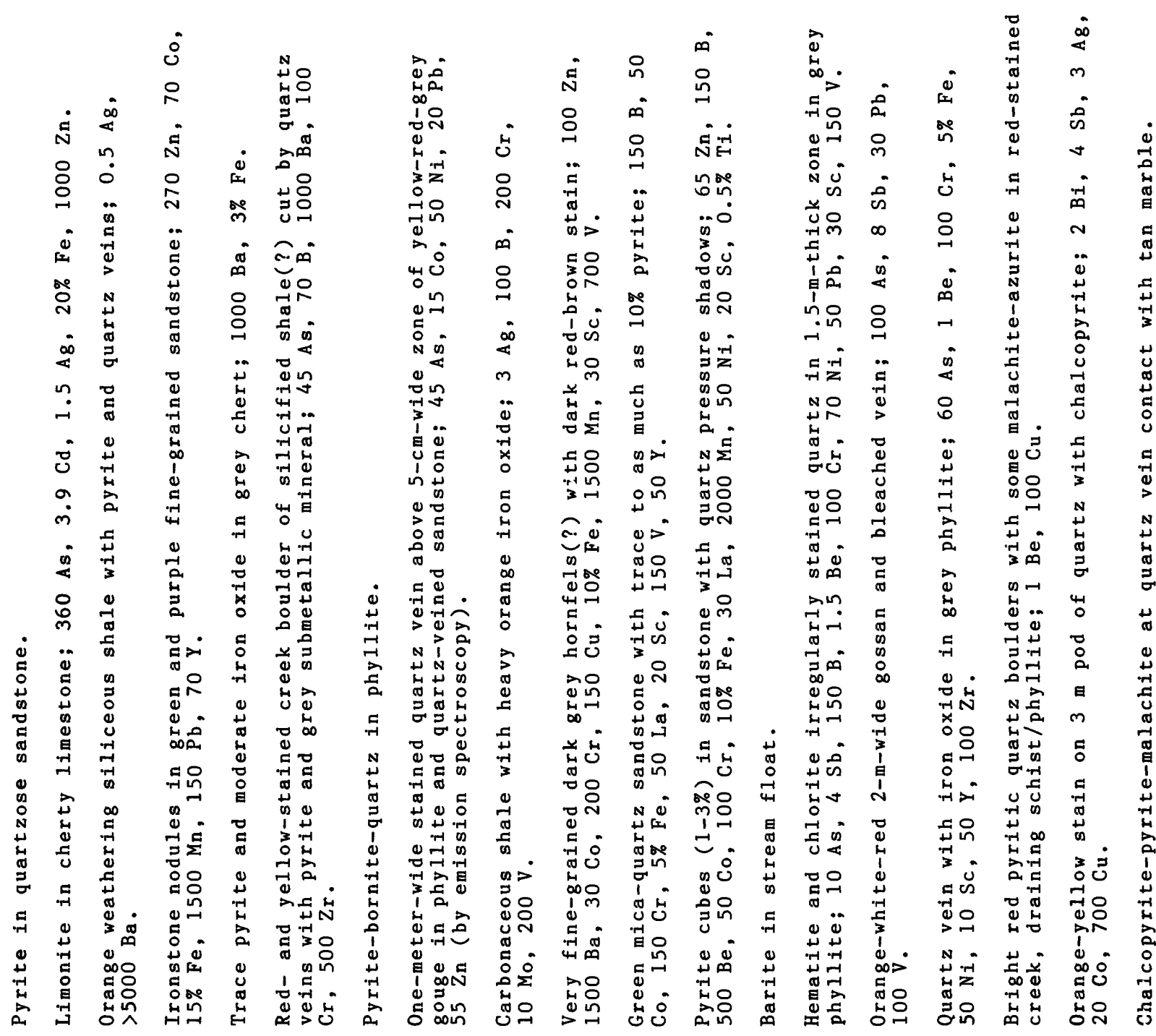

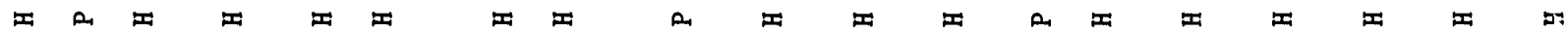

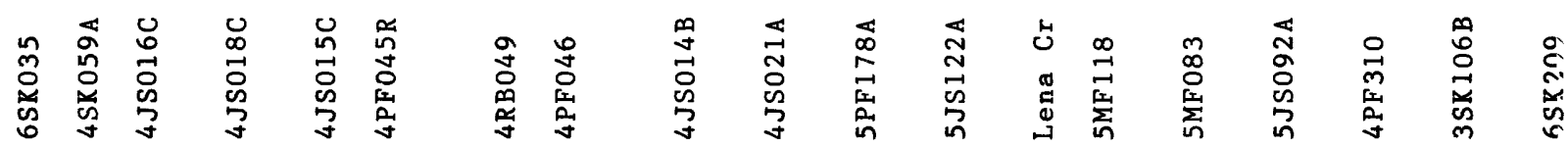

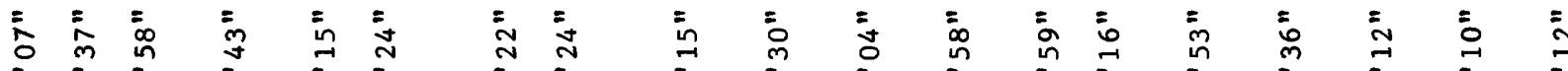

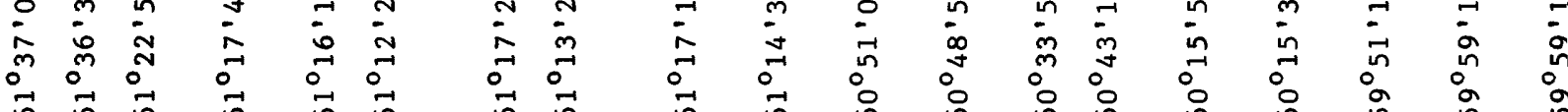

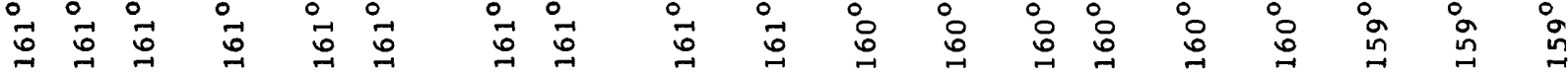

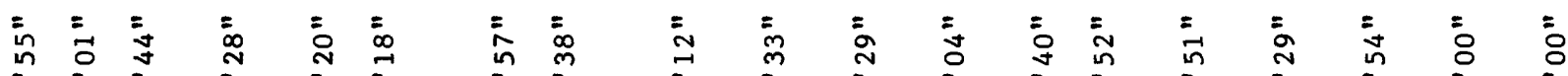

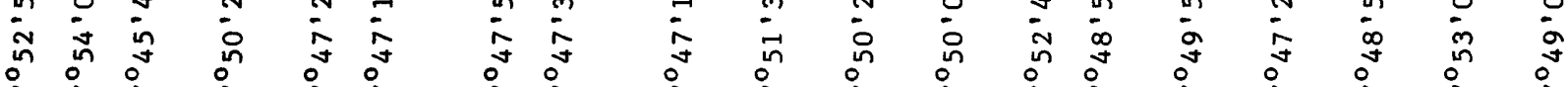

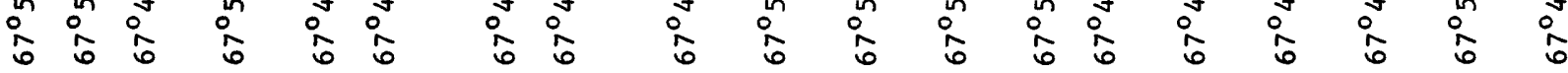

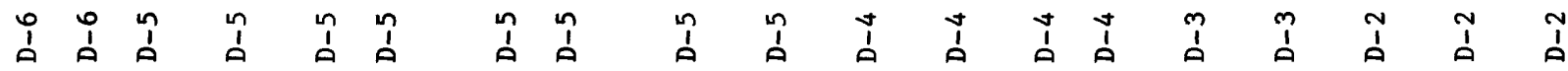

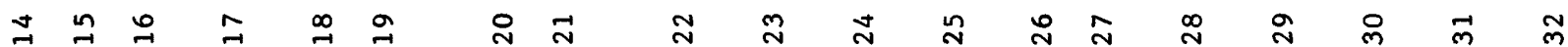




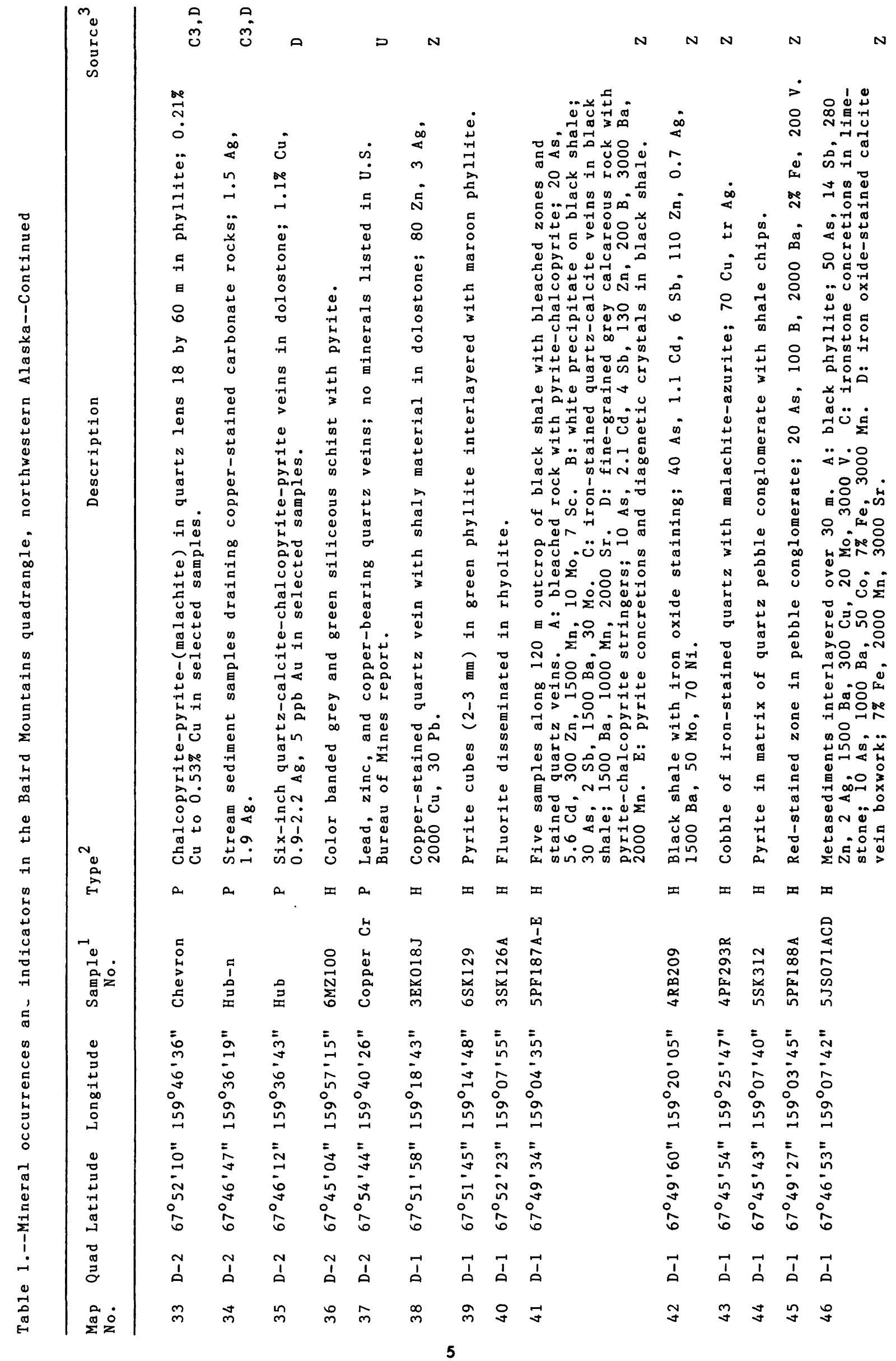




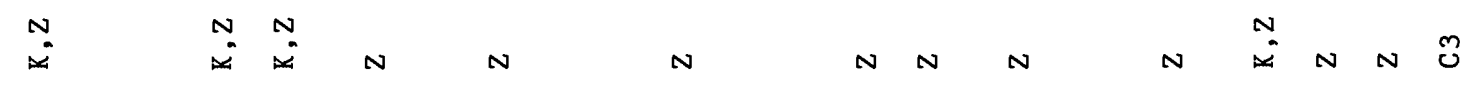

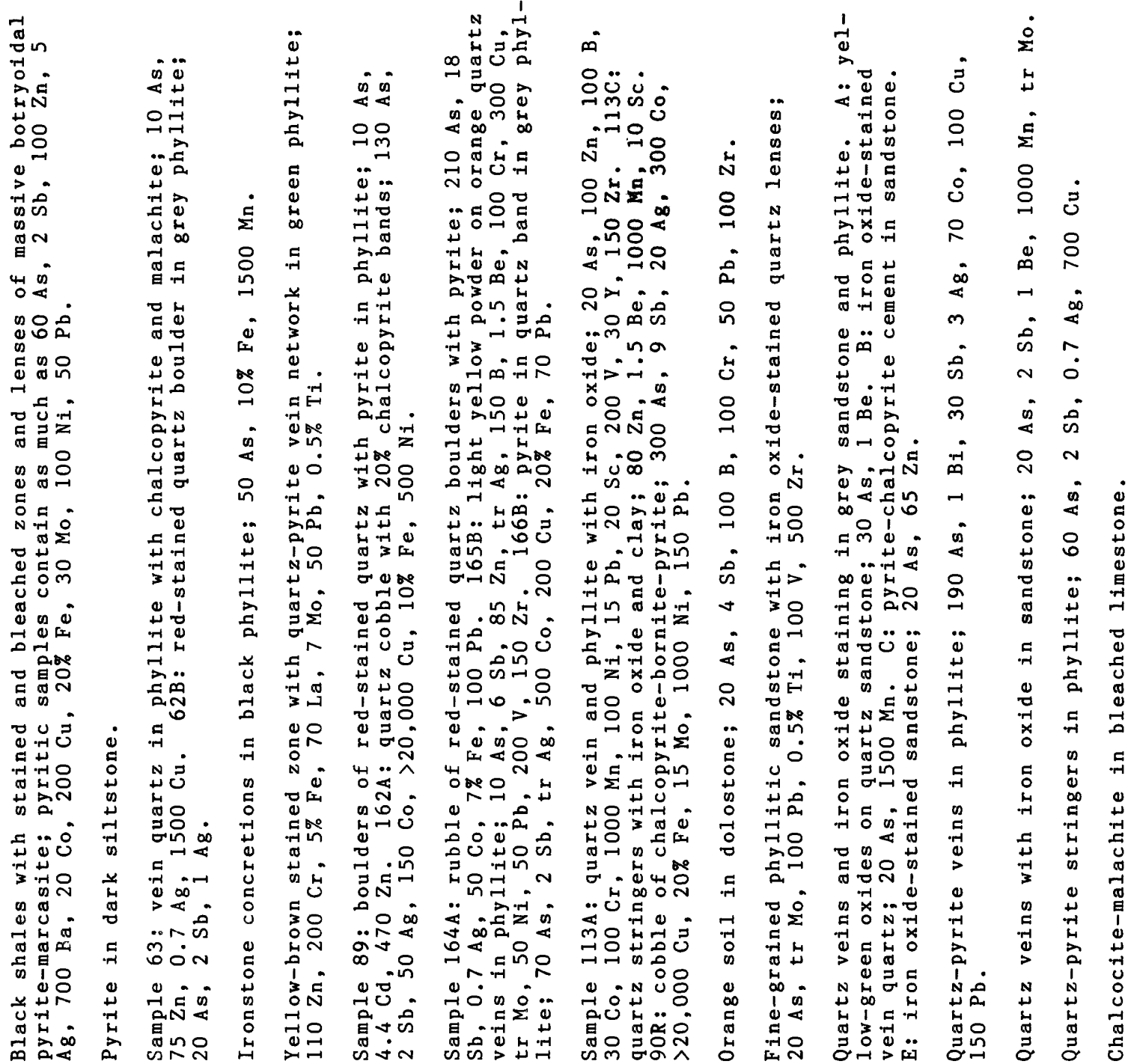

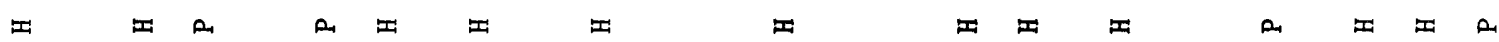

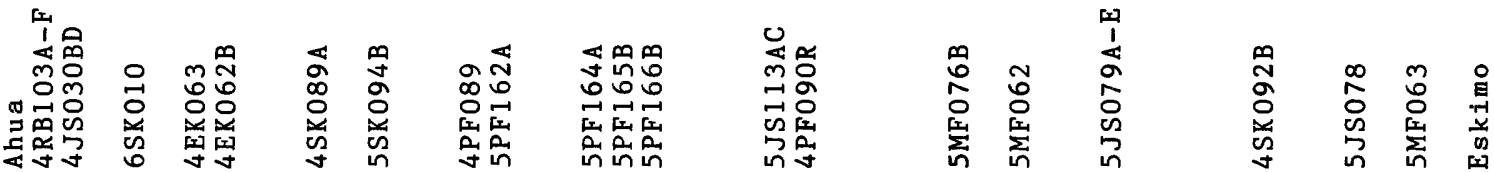

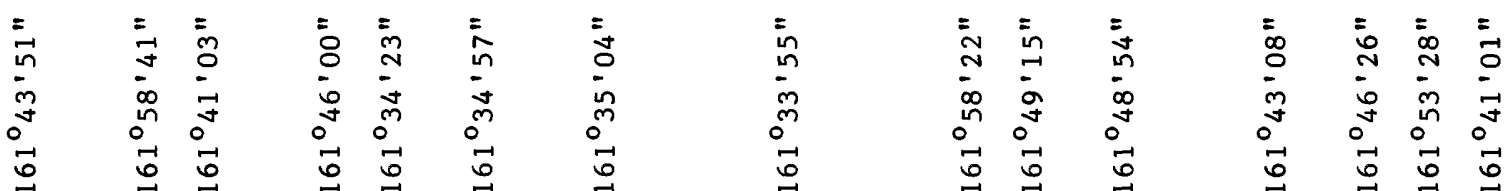

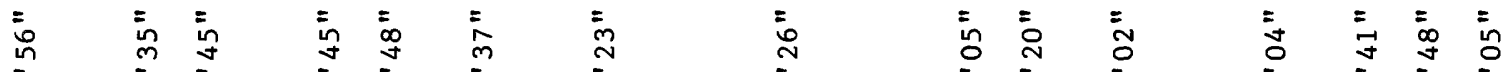

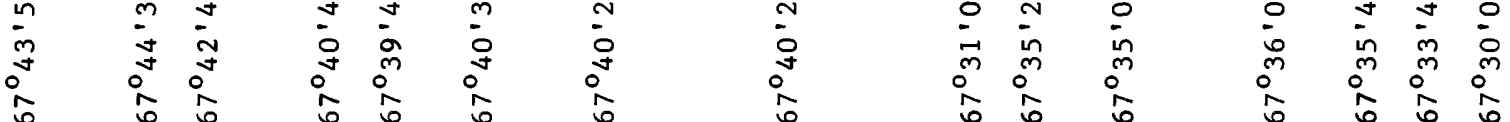

$\begin{array}{llllllllllllllll}1 & 0 & 0 & 0 & 0 & 0 & 0 & 0 & 0 & 0 & 0 & 0 & 0 & 0 & 0 \\ 0 & 0 & 1 & 1 & 1 & 1 & 1 & 1 & 1 & 1 & 1 & 1 & 1 & 1 & 1 & 0\end{array}$

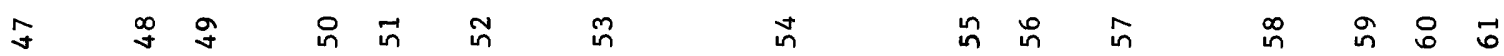




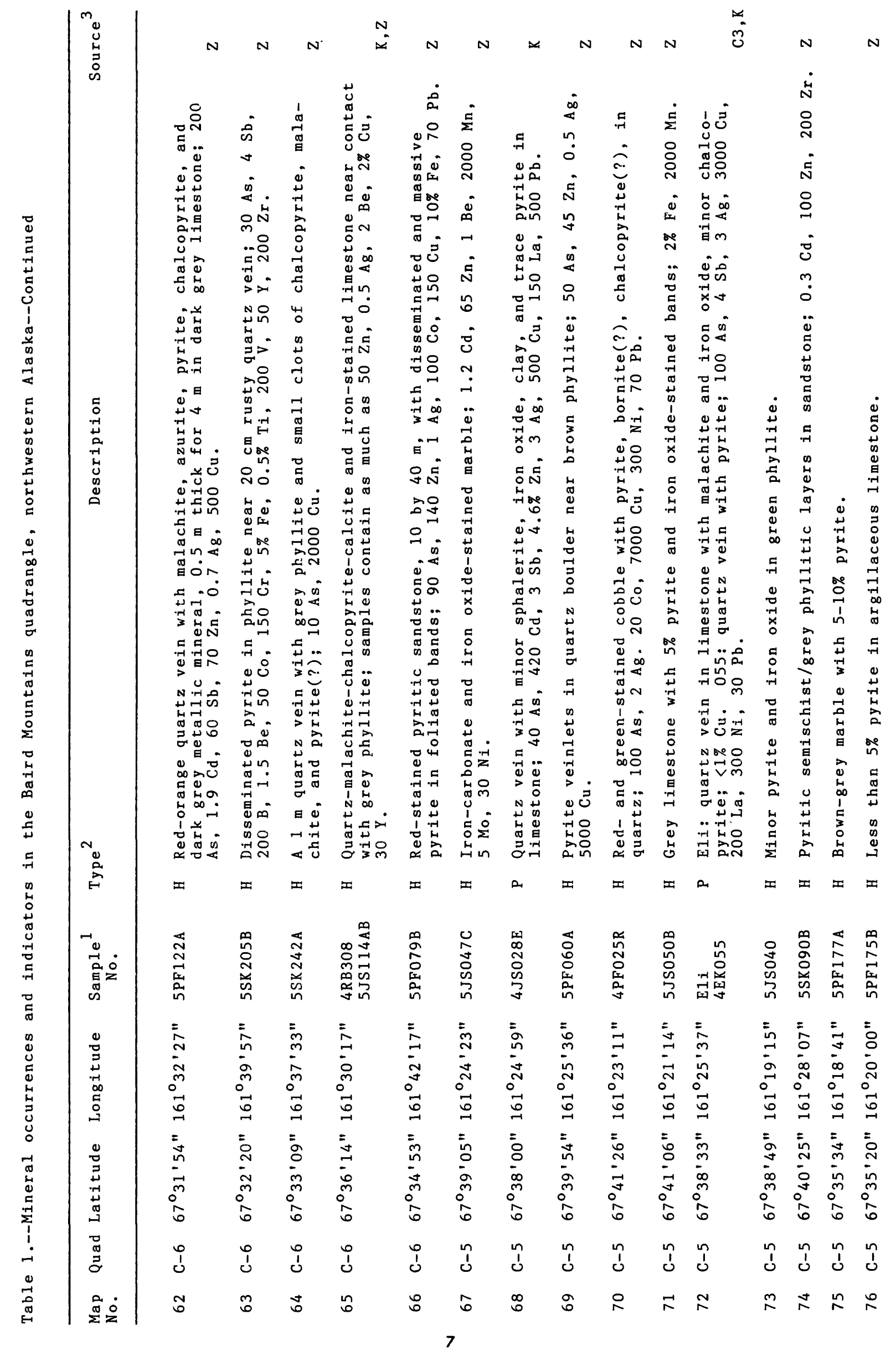




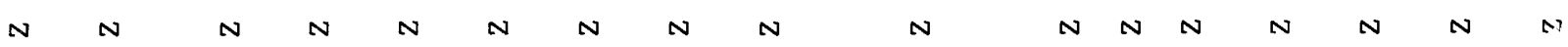

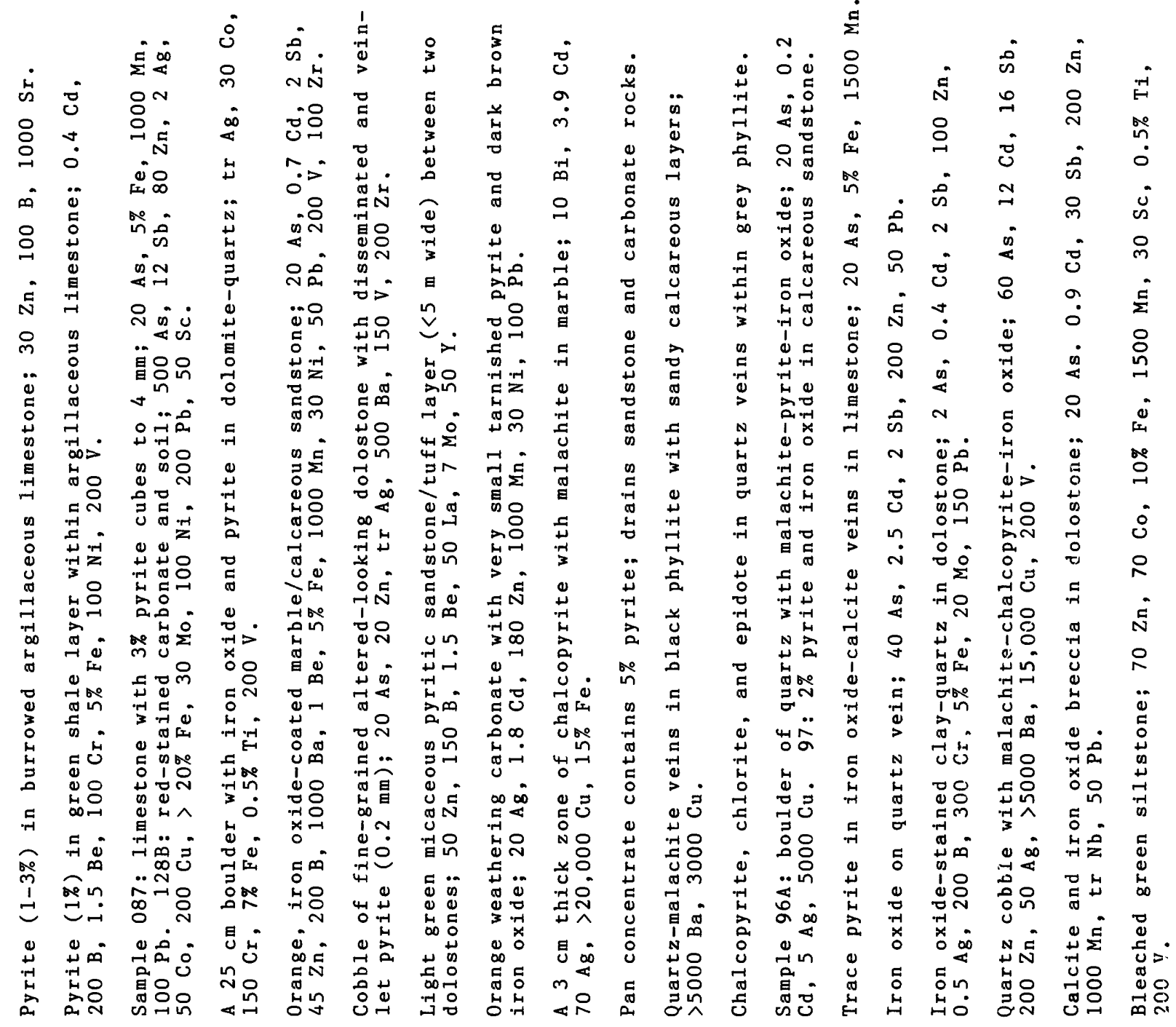

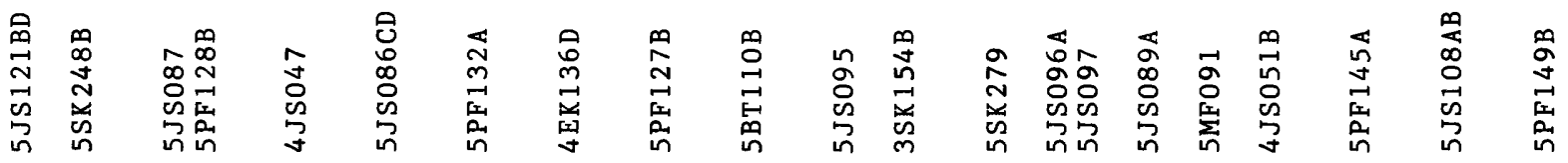

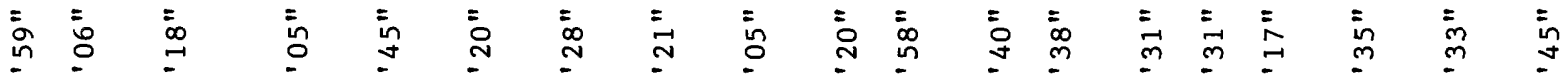

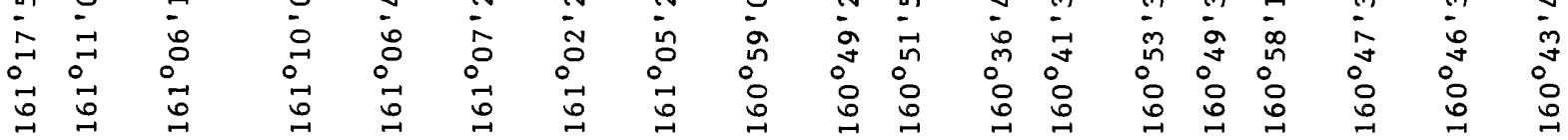

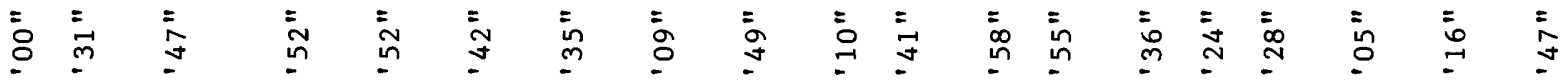

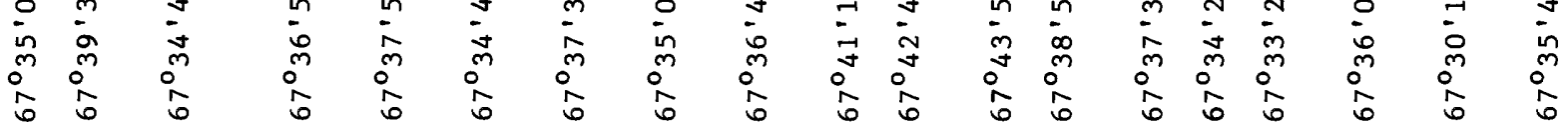

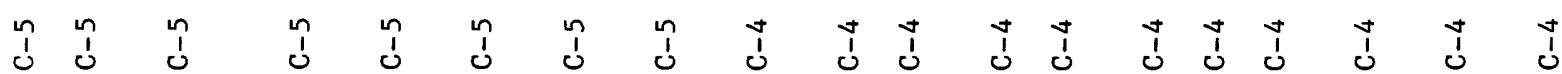

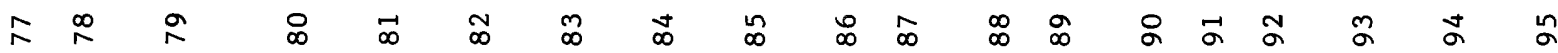




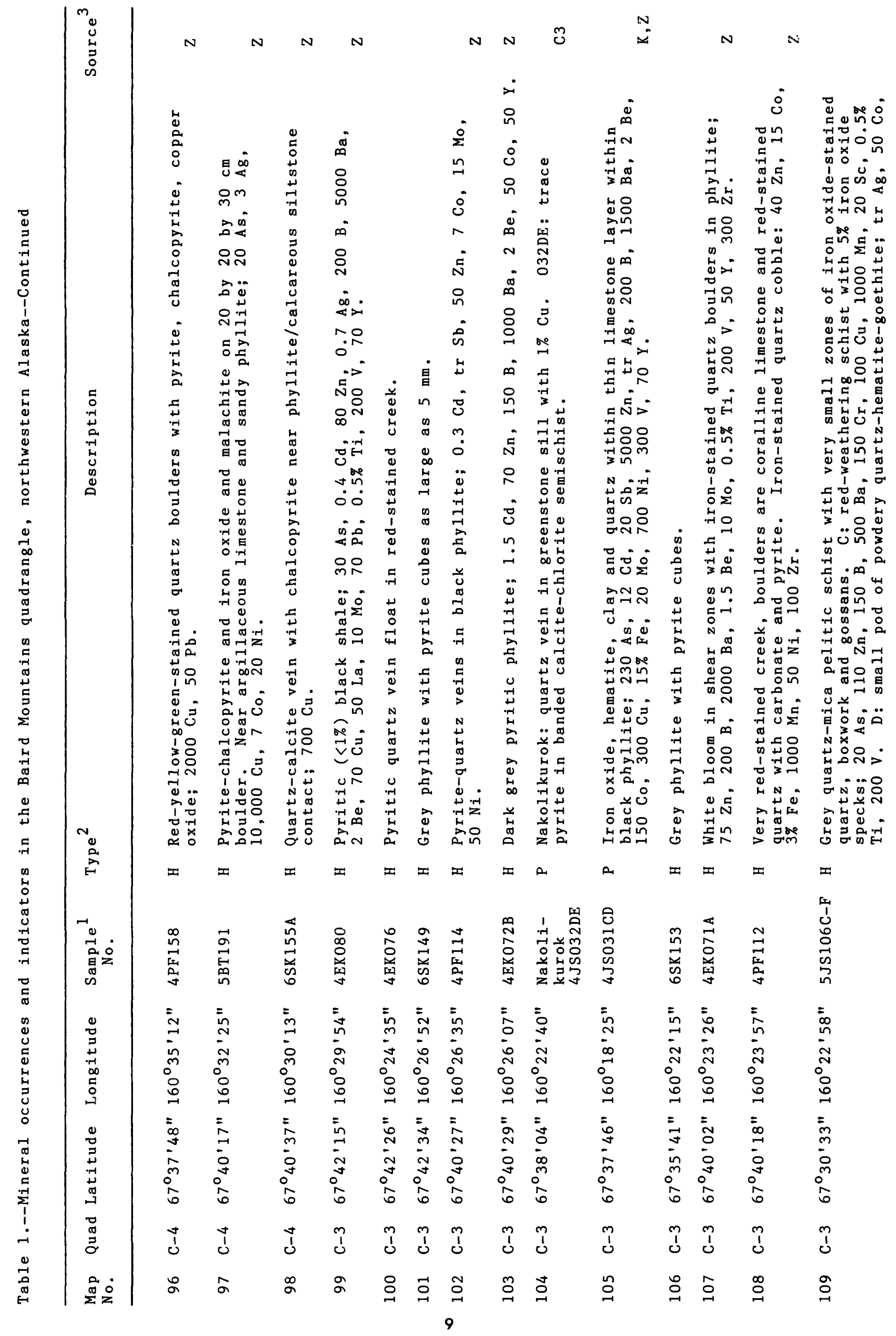


$N$ N N N N

$\therefore \quad n N a$

$\dot{\infty}$

Did

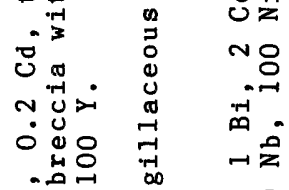

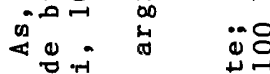

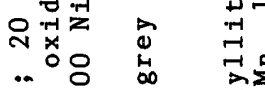

푸응

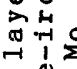

荡

兵出

论

Uñ

要离

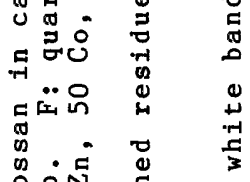

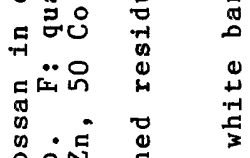

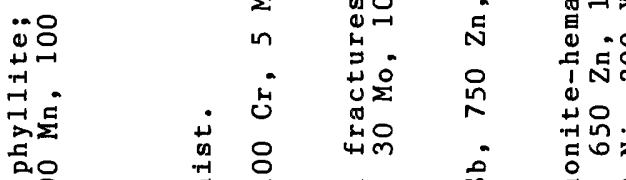

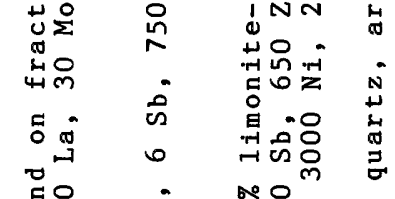

$\bigcup_{\substack{0 \\ \hdashline}}^{\infty}$

ตั

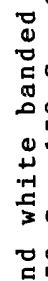

$\rightarrow 0^{n}$

a $-\infty$

武要

유윰

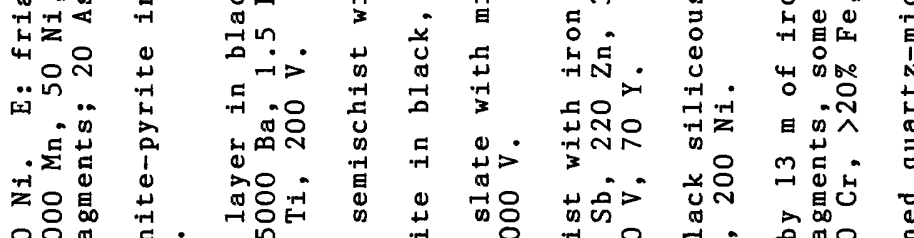

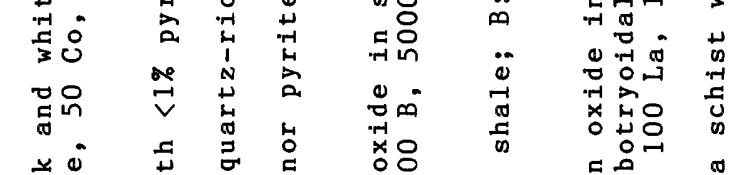

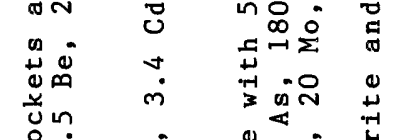

Un

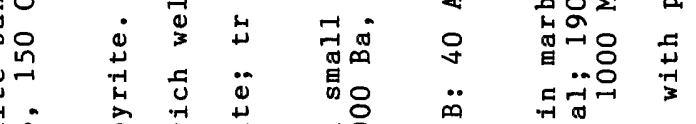

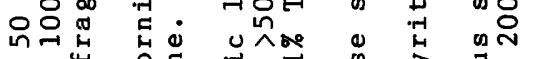

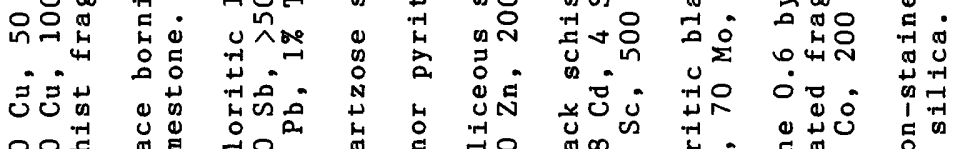

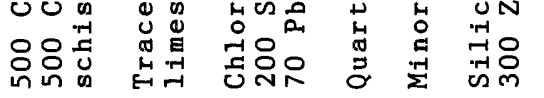

잉

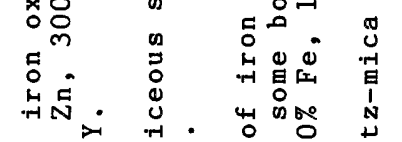

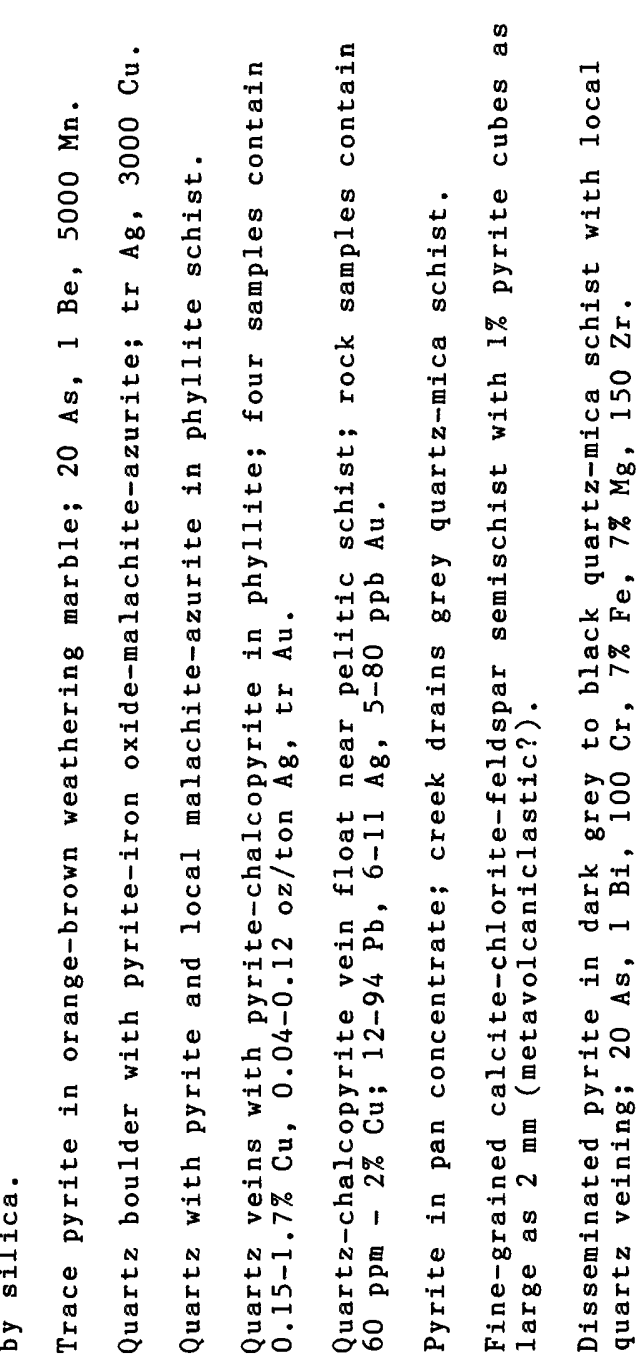

$\Sigma$

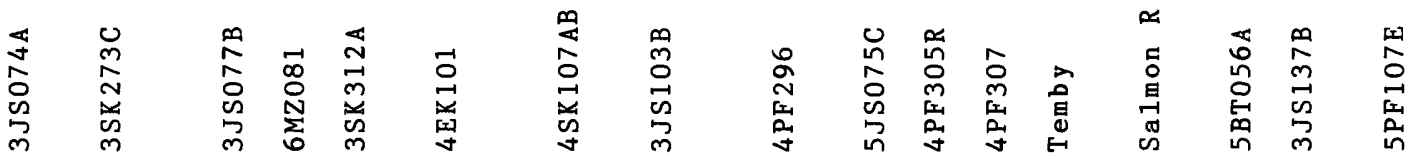

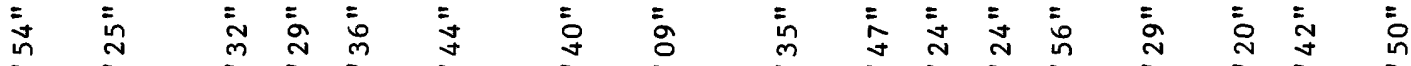

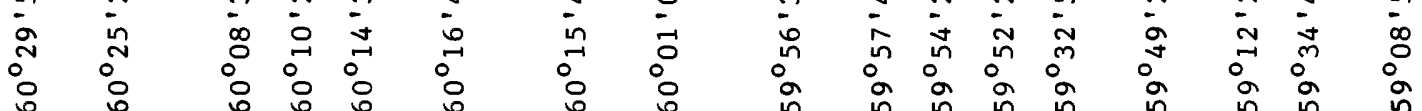

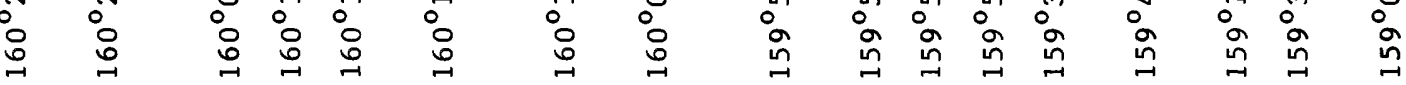

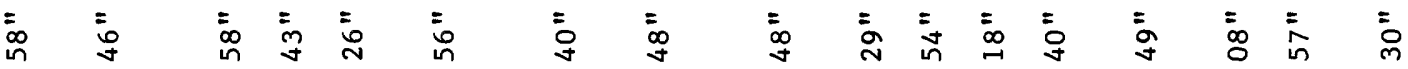

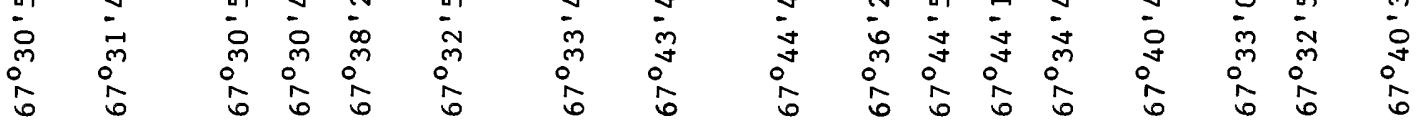

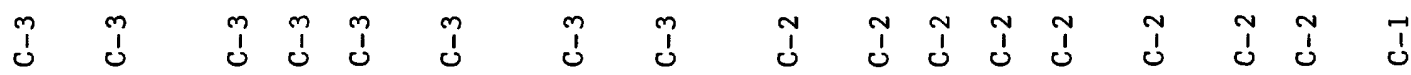

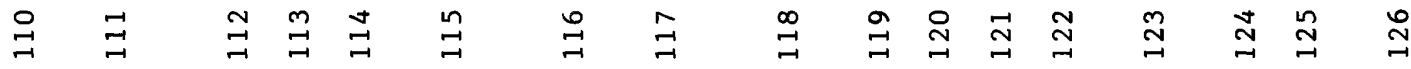




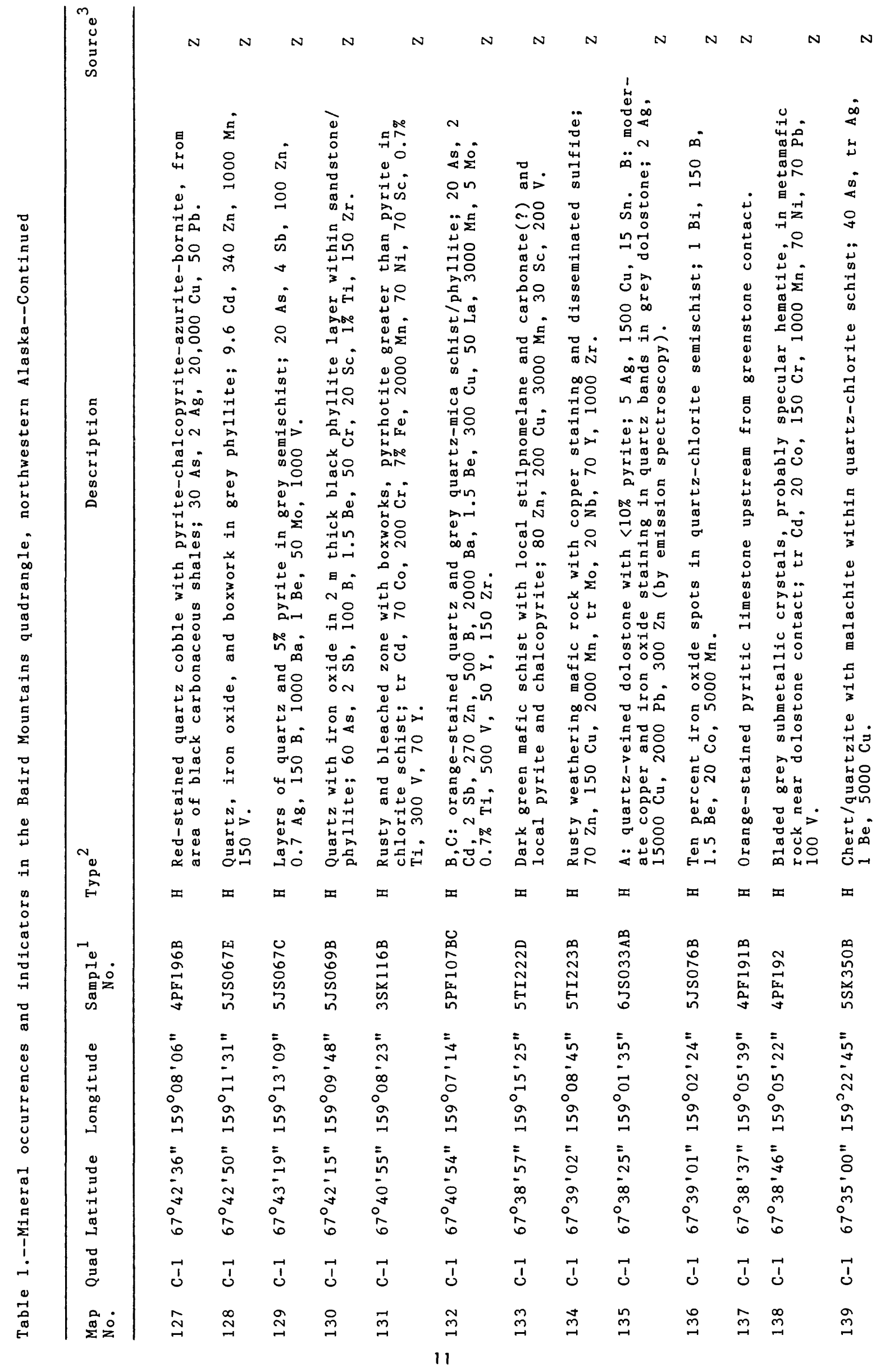




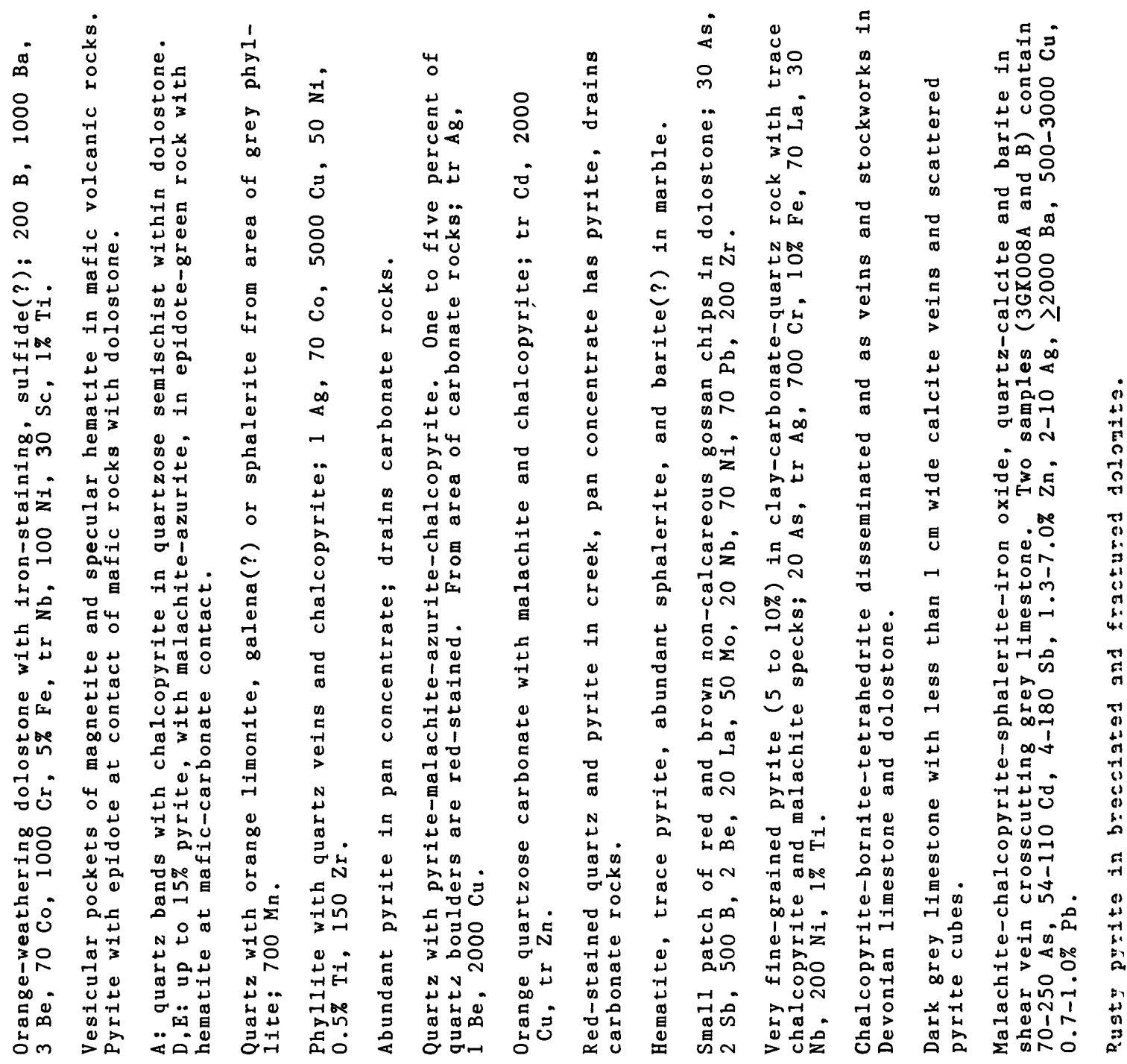

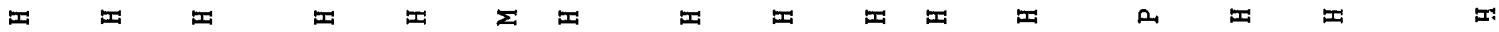

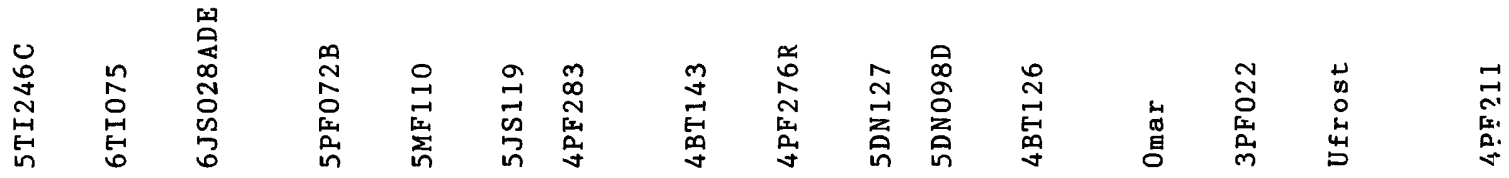

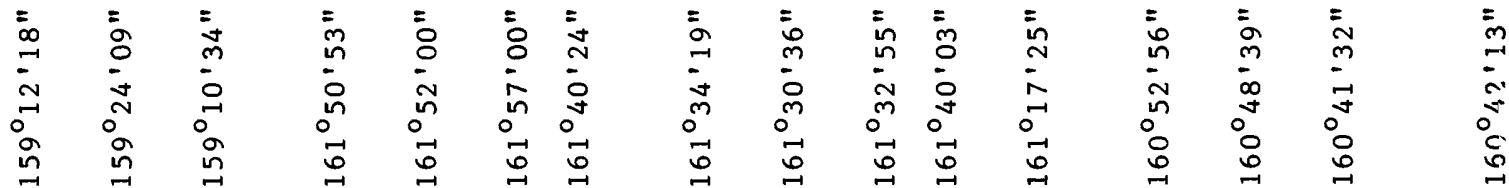

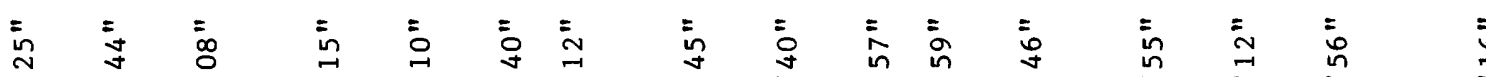

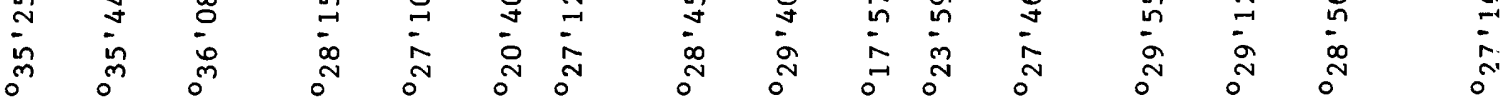

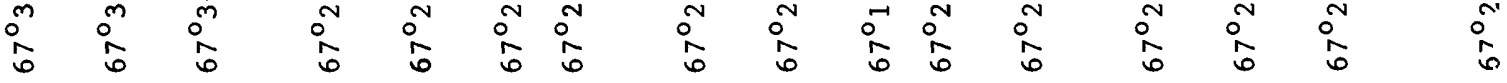

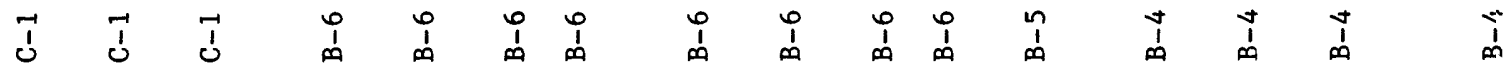

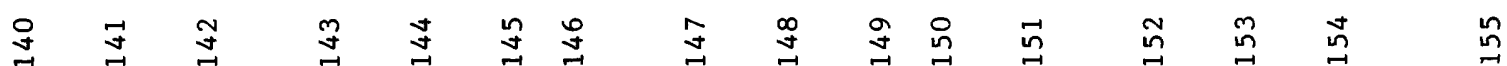




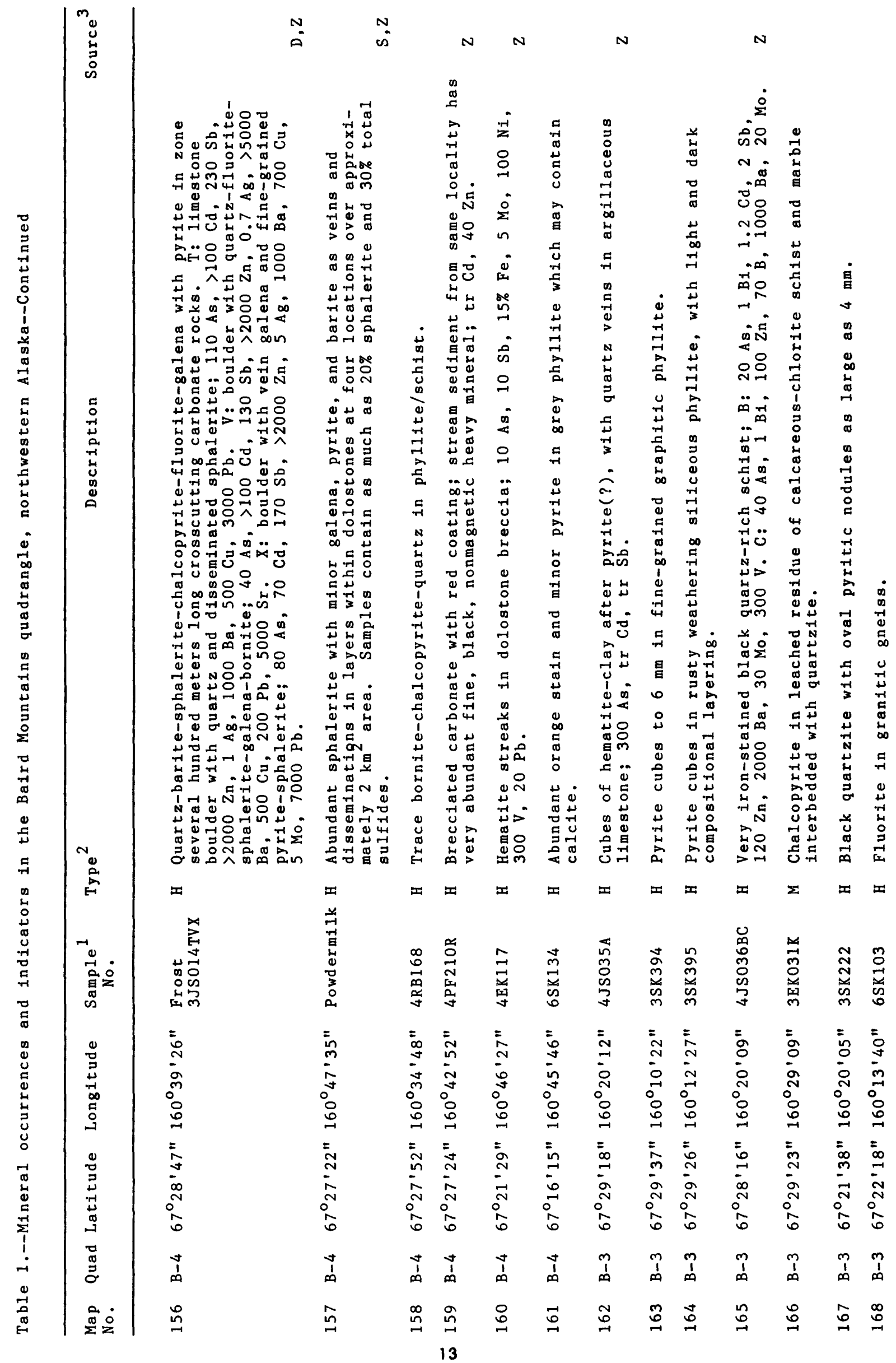




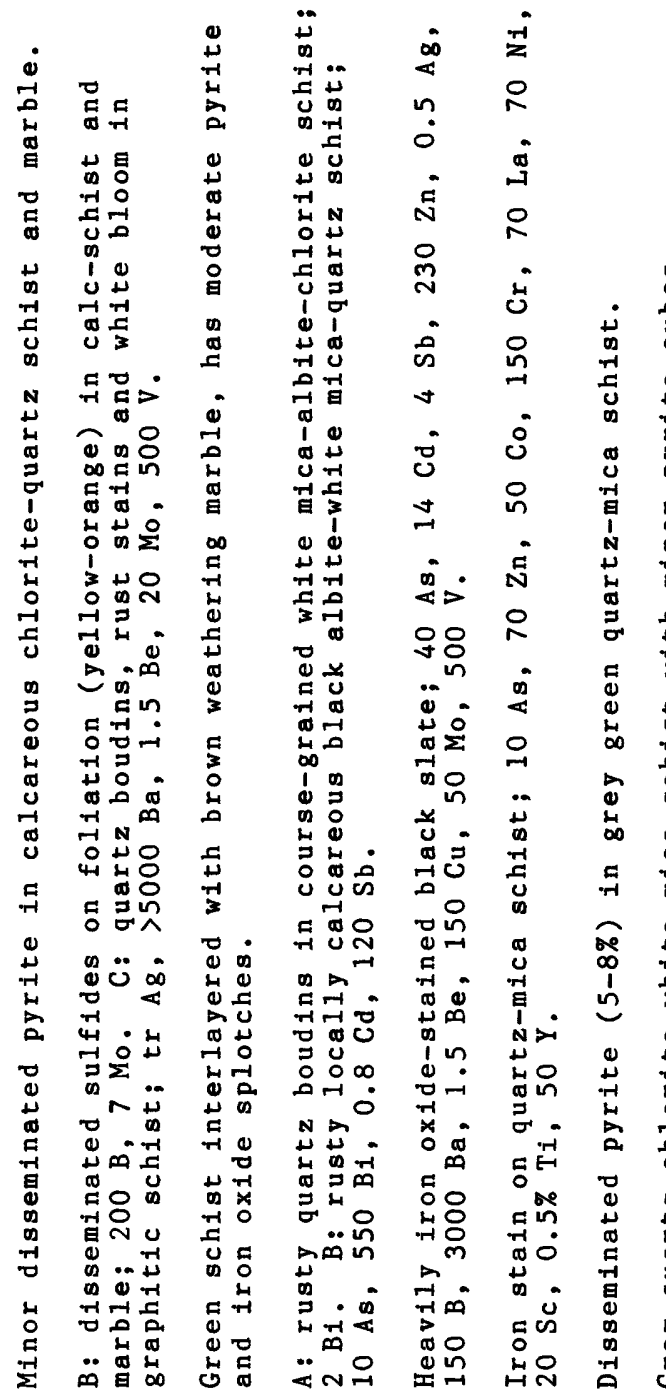

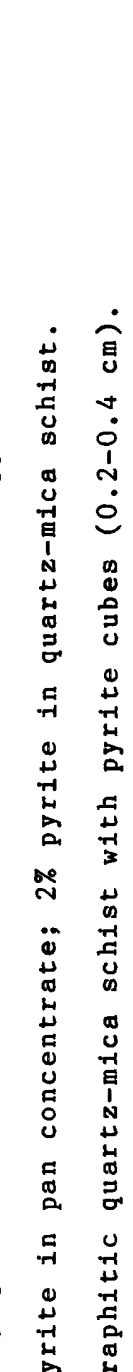

它

2ूषण

iै

我电曲

is

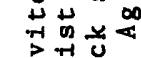

过皆出

当为的

其出号是

N⿴囗十

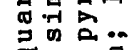

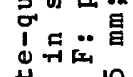

ज政

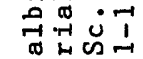

㟧的

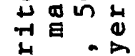

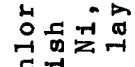

던 귱용

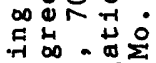

1..

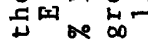

ॠ.

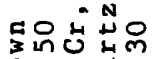

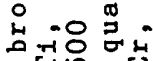

Hive

in $-\infty$

ลं0용

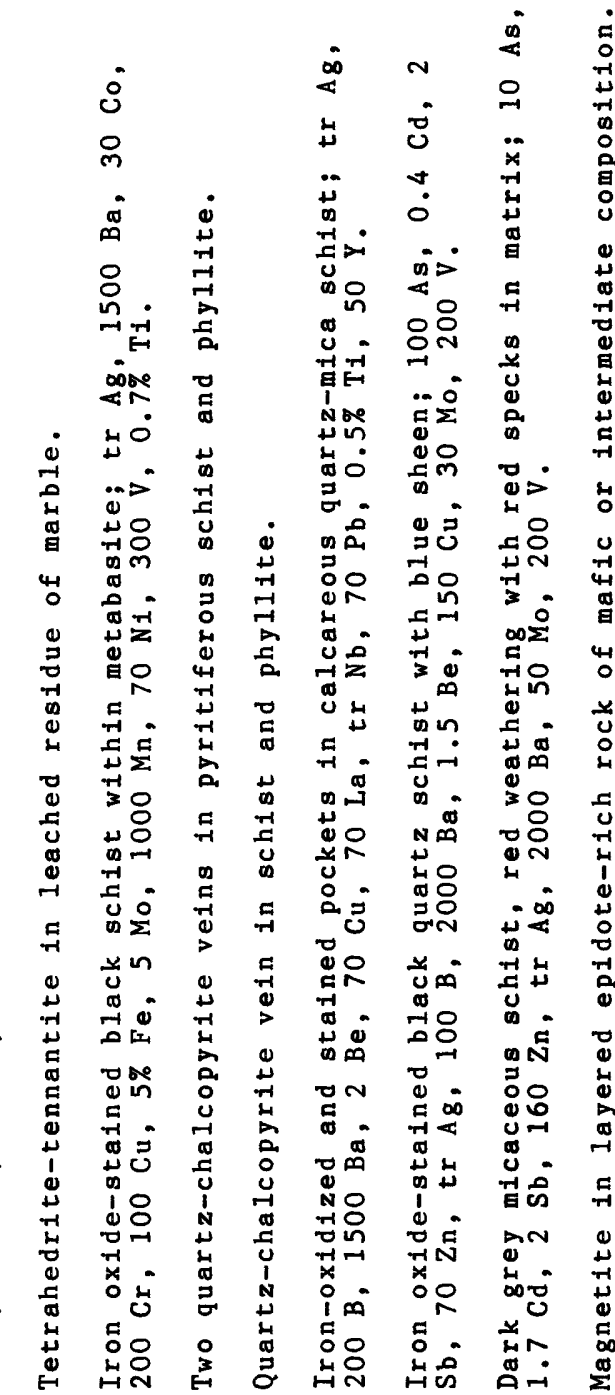

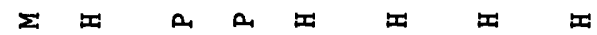

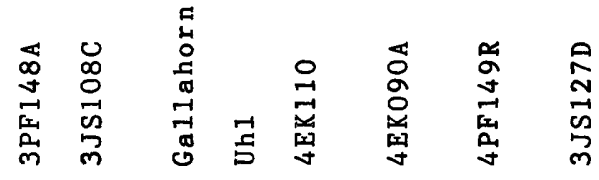

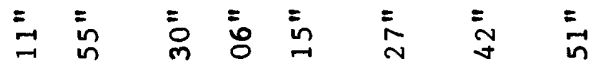

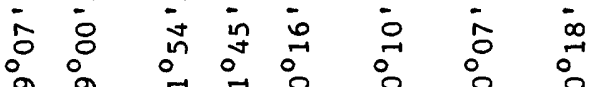

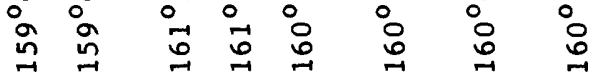
in

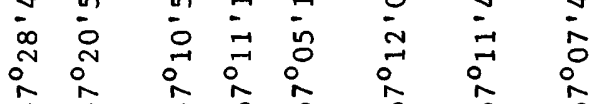

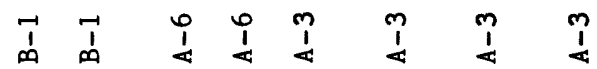

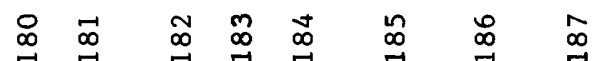




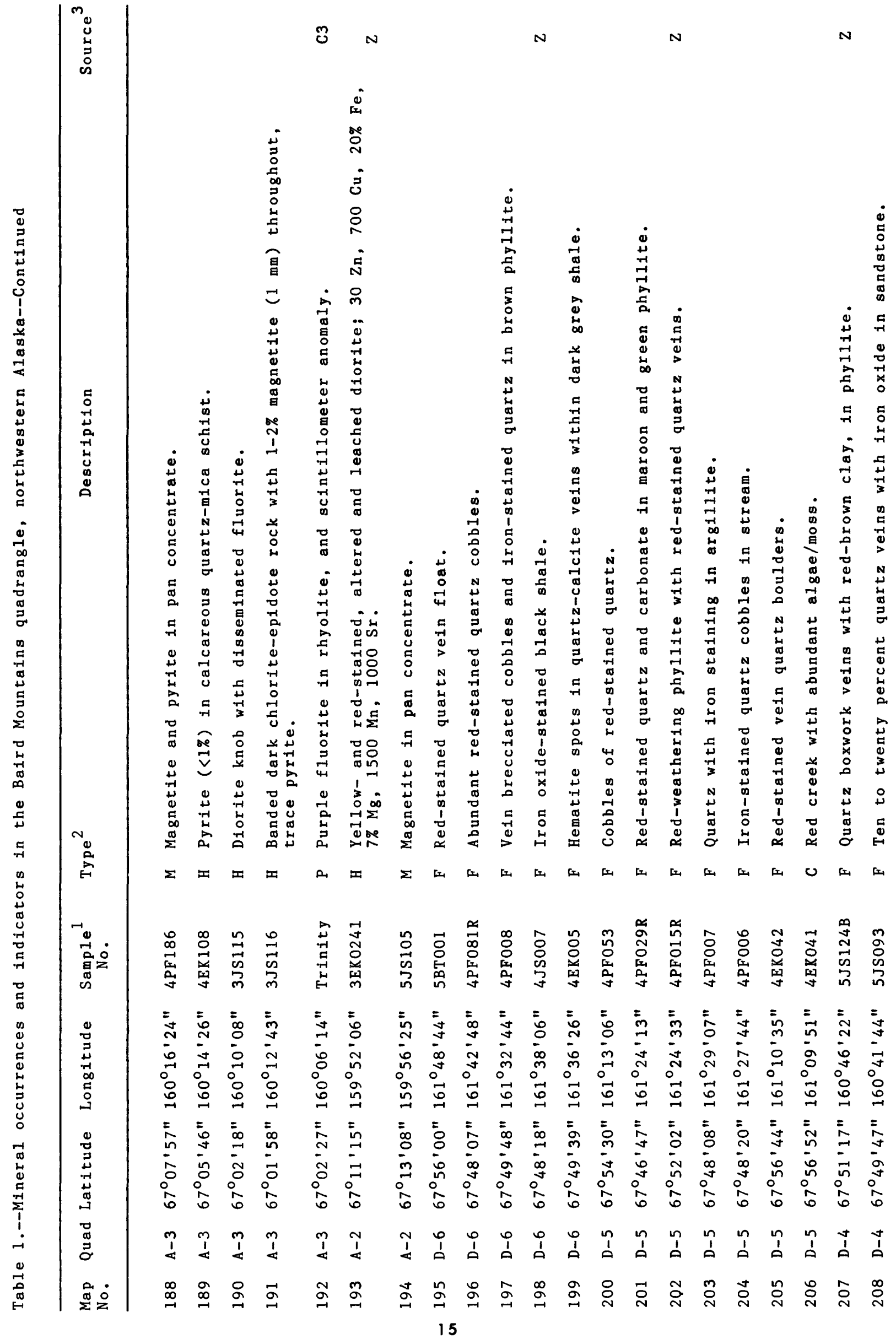




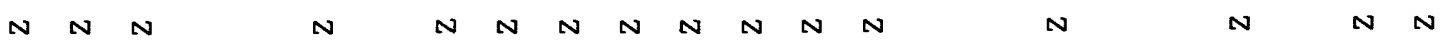

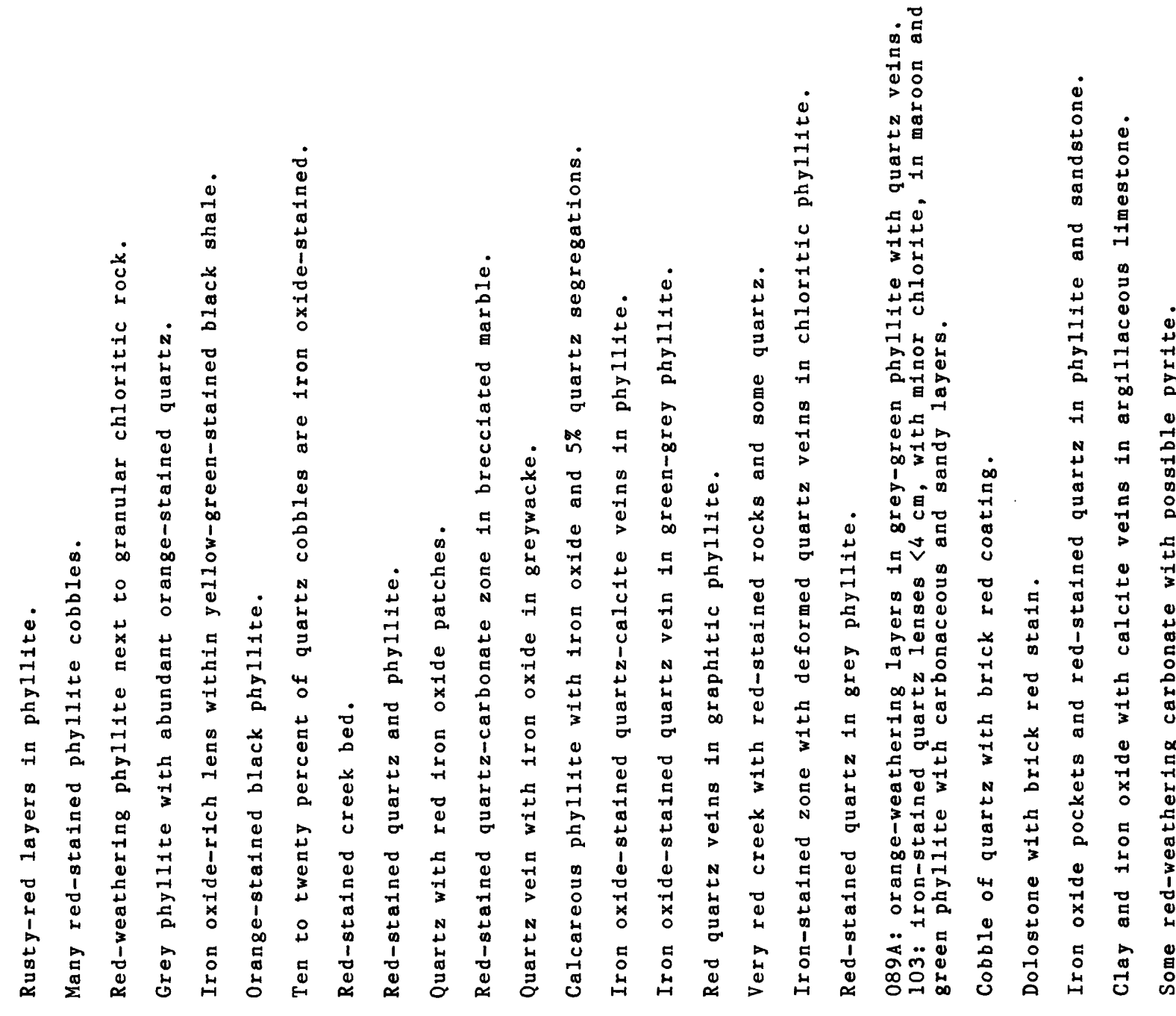

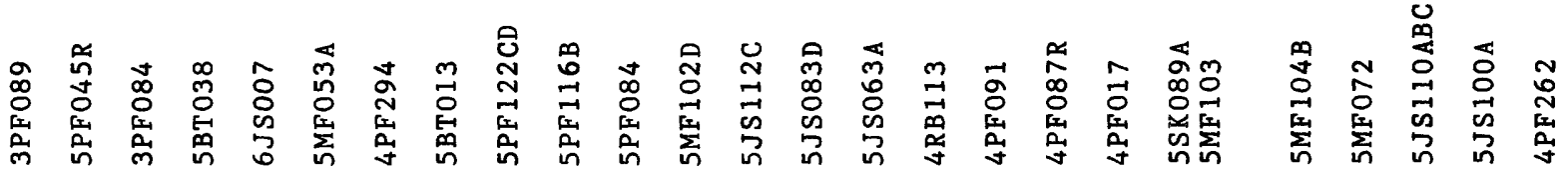

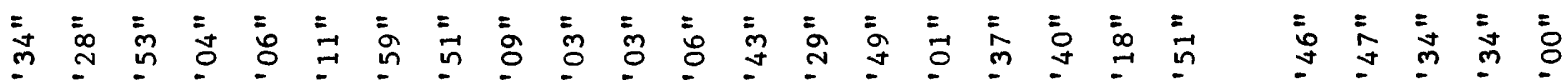

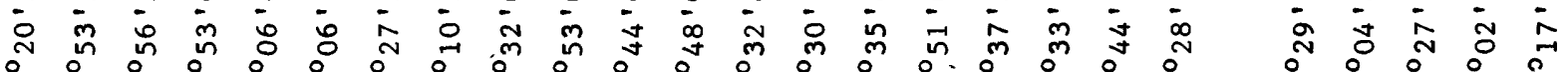

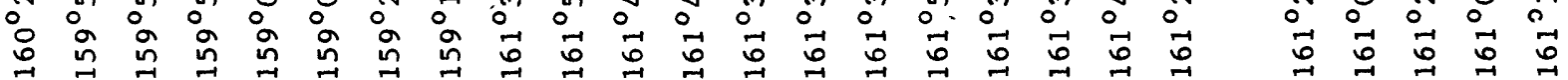

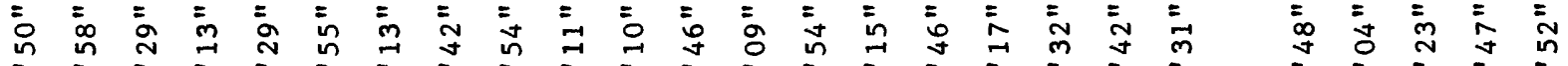

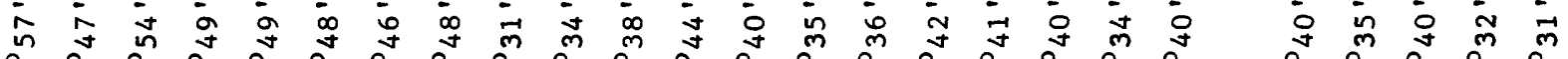

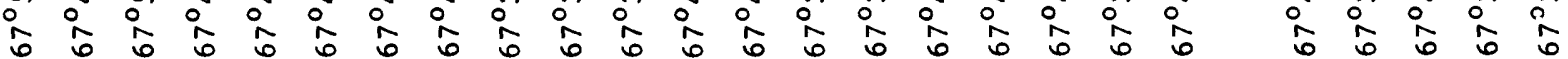

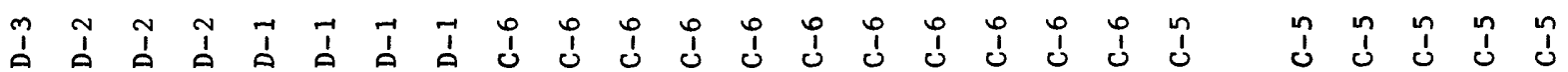

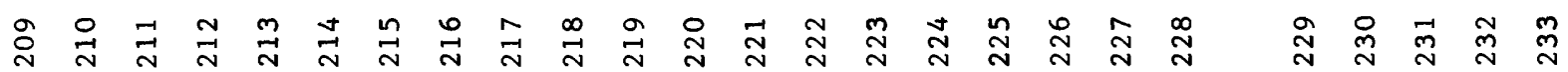




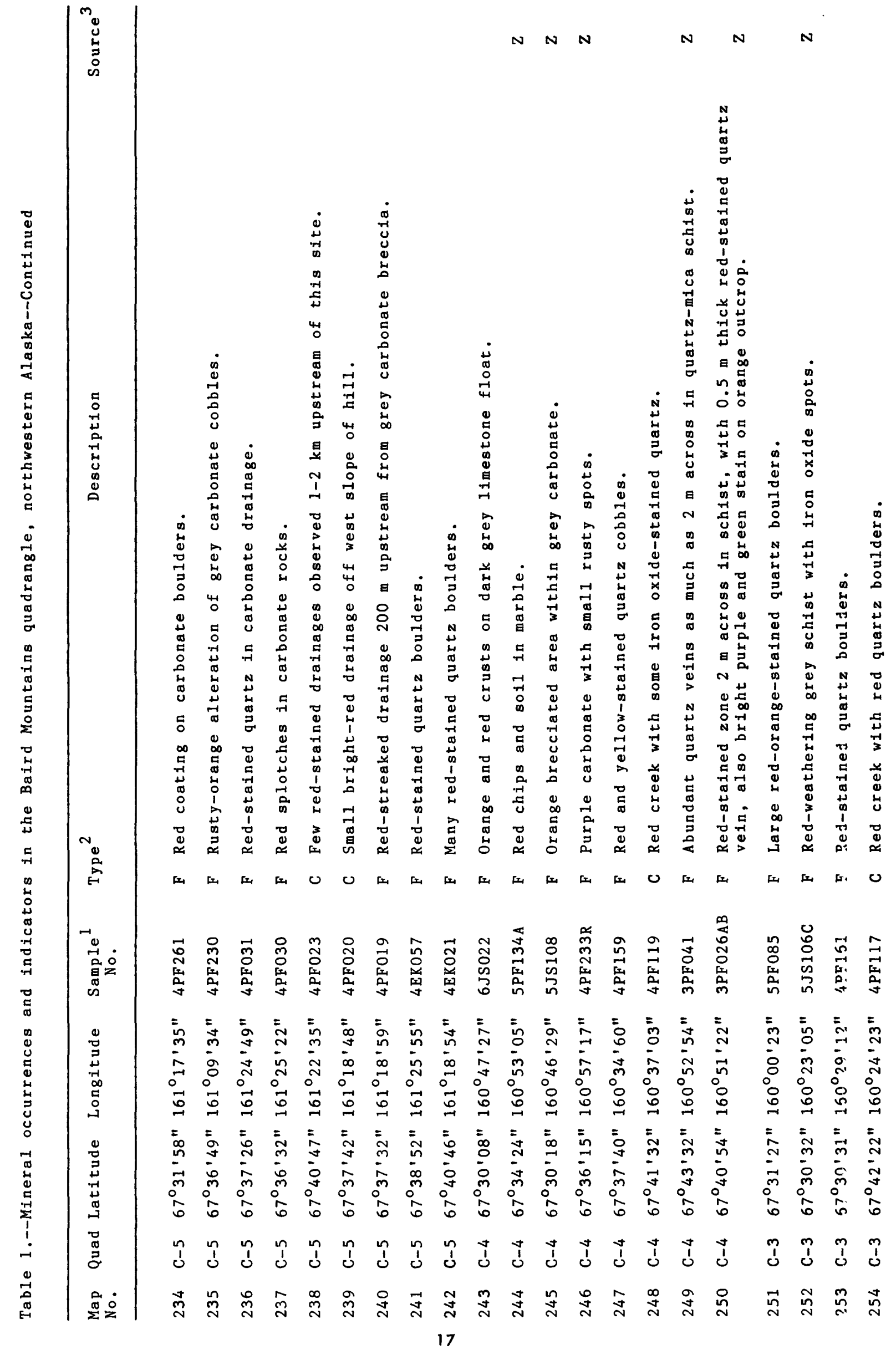



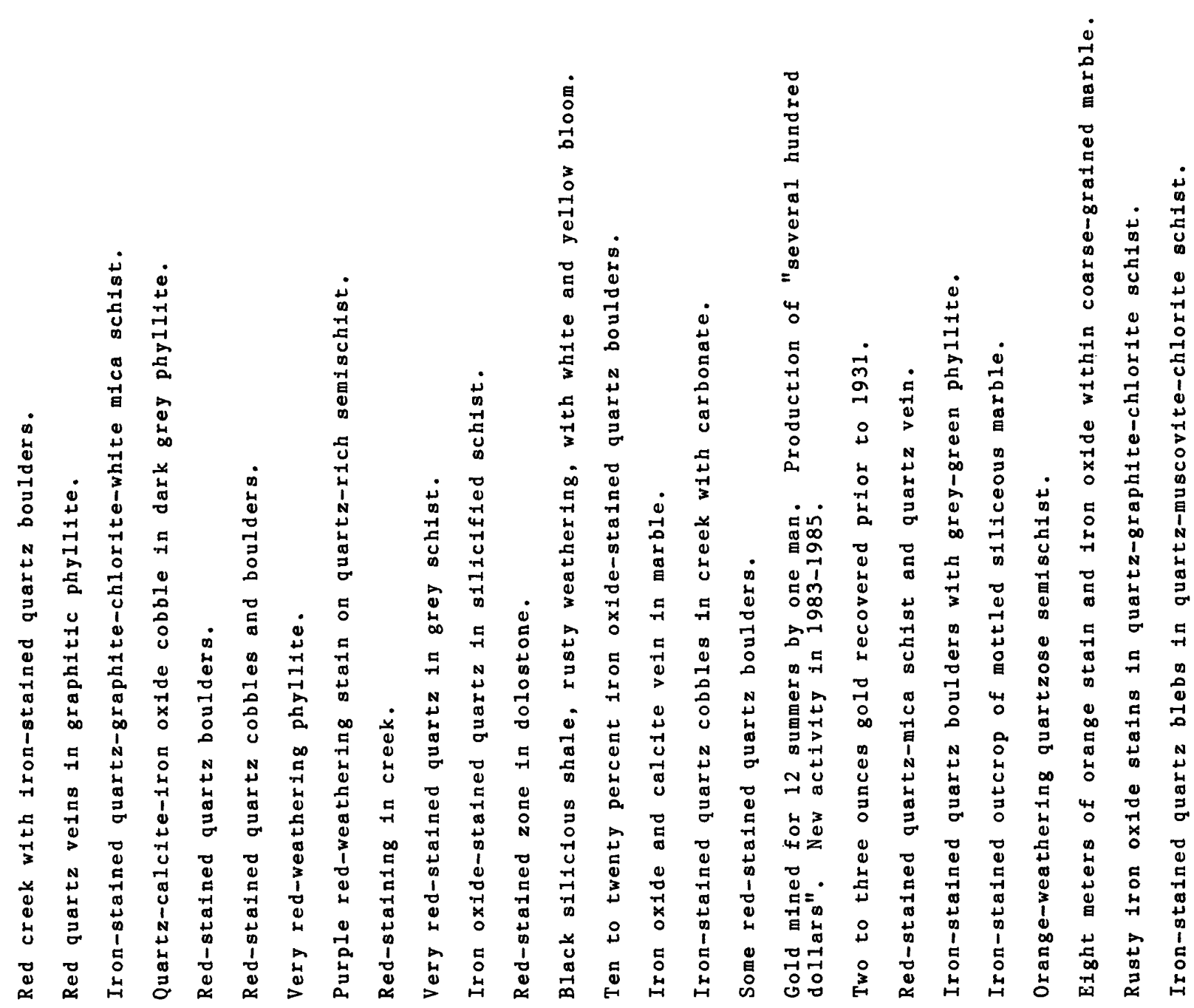

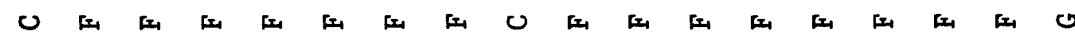

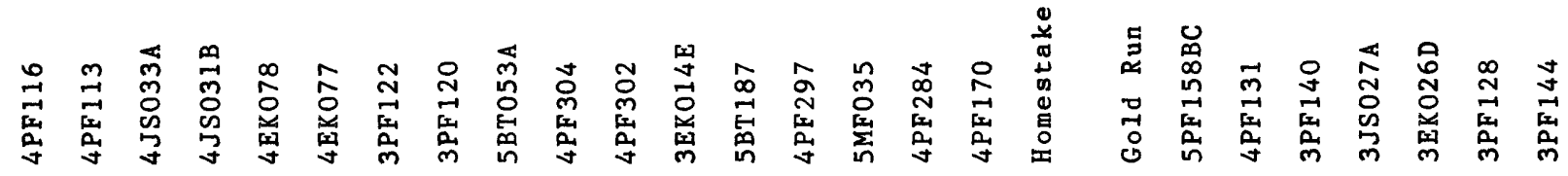

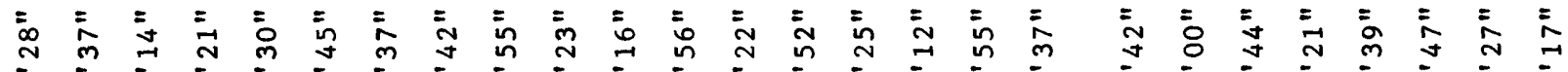

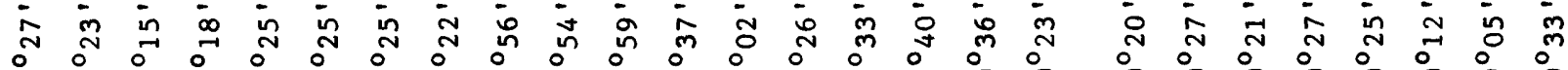

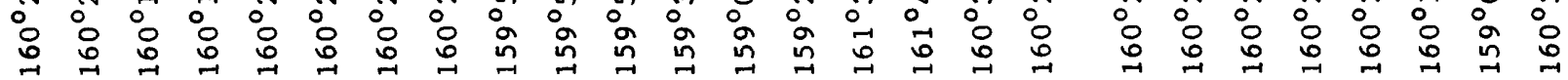

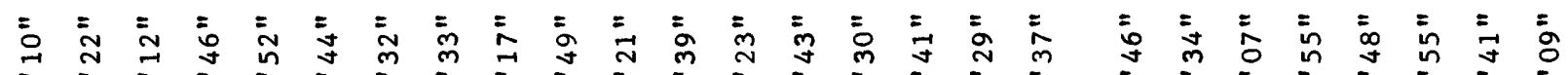

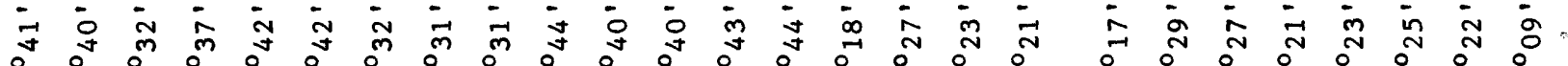

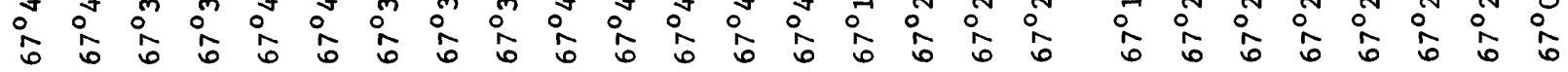

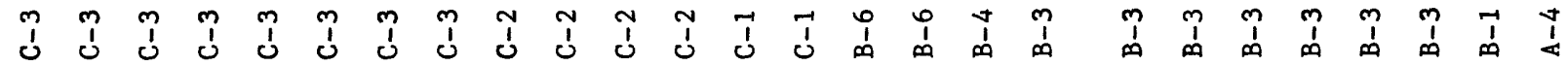

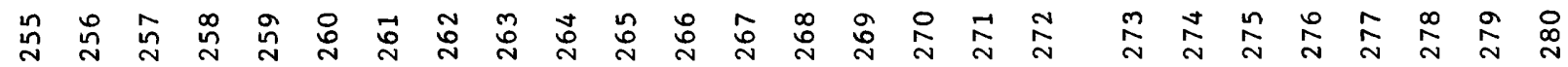




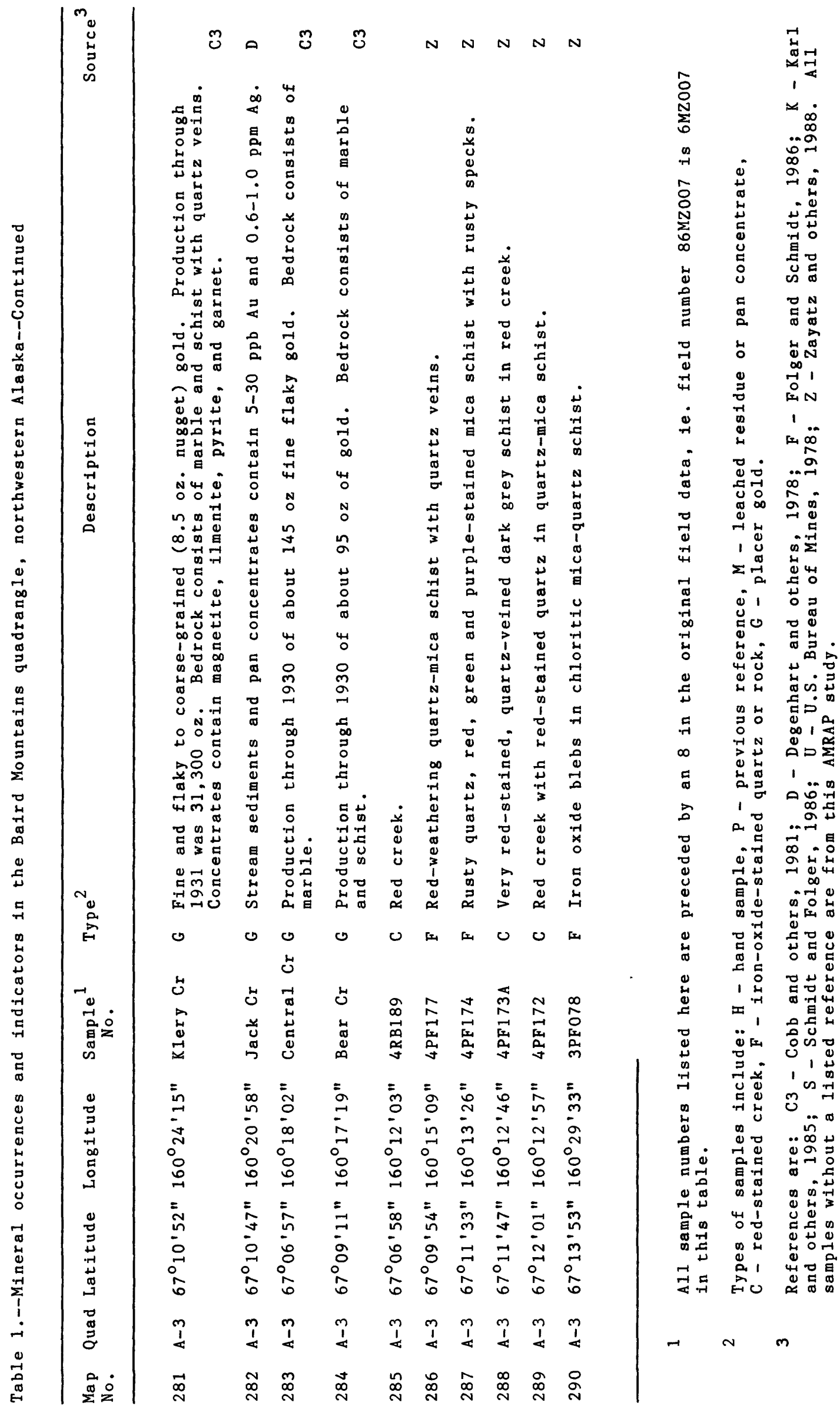


